\title{
ON D-PREINVEX-TYPE FUNCTIONS
}

\author{
JIAN-WEN PENG AND DAO-LI ZHU
}

Received 7 April 2006; Revised 10 July 2006; Accepted 26 July 2006

Some properties of $D$-preinvexity for vector-valued functions are given and interrelations among $D$-preinvexity, $D$-semistrict preinvexity, and $D$-strict preinvexity for vector-valued functions are discussed.

Copyright (c) 2006 J.-W. Peng and D.-L. Zhu. This is an open access article distributed under the Creative Commons Attribution License, which permits unrestricted use, distribution, and reproduction in any medium, provided the original work is properly cited.

\section{Introduction and preliminaries}

Convexity and generalized convexity play a central role in mathematical economics, engineering, and optimization theory. Therefore, the research on convexity and generalized convexity is one of the most important aspects in mathematical programming (see [1-4, 6-11] and the references therein). Weir and Mond [7] and Weir and Jeyakumar [6] introduced the definition of preinvexity for the scalar function $f: X \subset \mathbb{R}^{n} \rightarrow \mathbb{R}$. Recently, Yang and Li [9] gave some properties of preinvex function under Condition C. Yang and $\mathrm{Li}$ [9] introduced the definitions of strict preinvexity and semistrict preinvexity for the scalar function $f: X \subset \mathbb{R}^{n} \rightarrow \mathbb{R}$ and discussed the relationships among preinvexity, strictly preinvexity, and semistrictly preinvexity for the scalar functions. Yang [8] also obtained some properties of semistrictly convex function and discussed the interrelations among convex function, semistrictly convex function, and strictly convex function.

Throughout this paper, we will use the following assumptions. Let $X$ be a real topological vector space and $Y$ a real locally convex vector space, let $S \subset X$ be a nonempty subset, let $D \subset Y$ be a nonempty pointed closed convex cone, $Y^{*}$ is the dual space of $Y$, equipped with the weak* topology. The dual cone $D^{*}$ of cone $D$ is defined by

$$
D^{*}=\left\{f \in Y^{*}: f(y)=\langle f, y\rangle \geq 0, \forall y \in D\right\} .
$$

From the bipolar theorem, we have the following.

Lemma 1.1. For all $q \in D^{*}, q(d) \geq 0$ if and only if $d \in D$. 
As a generalization of the definition of preinvexity for real-valued functions, Kazmi [3] introduced the definition of $D$-preinvexity for vector-valued functions as follows.

Definition 1.2 (see $[6,7]$ ). A set $S \subset X$ is said to be invex if there exists a vector function $\eta: X \times X \rightarrow X$ such that

$$
x, y \in S, \quad \alpha \in[0,1] \Longrightarrow y+\alpha \eta(x, y) \in S .
$$

Definition 1.3 (see [3]). Let $S \subset X$ be an invex set with respect to $\eta: X \times X \rightarrow X$. The vector-valued function $F: S \rightarrow Y$ is said to be $D$-preinvex on $S$ if for all $x, y \in S, \alpha \in(0,1)$, one has

$$
F(y+\alpha \eta(x, y)) \in \alpha F(x)+(1-\alpha) F(y)-D
$$

Equivalently, (1.3) can be written as

$$
\alpha F(x)+(1-\alpha) F(y)-F(y+\alpha \eta(x, y)) \in D
$$

In [3], Kazmi showed that (i) if $F: S \rightarrow Y$ is $D$-preinvex, then any local weak minimum of $F$ is a global weak minimum; (ii) if $F: S \rightarrow Y$ is $D$-preinvex and Fréchet differentiable, then the vector optimization problem $\min _{x \in S} F(x)$ and the vector variational-like inequality

$$
\left\langle F^{\prime}\left(x_{0}\right), \eta\left(x, x_{0}\right)\right\rangle \notin-\operatorname{int} D, \quad \forall x \in S,
$$

have the same solutions, where $F^{\prime}\left(x_{0}\right)$ is the Fréchet derivative of $F$ at $x_{0}$.

In [1], Bhatia and Mehra introduced the definition of $D$-preinvexity for set-valued functions and obtained some Lagrangian duality theorems for set-valued fractional program.

As generalizations of definitions of strict preinvexity and semistrict preinvexity for scalar function, we introduce the definitions of $D$-strict preinvexity and $D$-semistrict preinvexity for vector-valued functions as follows.

Definition 1.4. Let $S \subset X$ be an invex set with respect to $\eta: X \times X \rightarrow X$. The vector-valued function

(i) $F: S \rightarrow Y$ is said to be $D$-semistrictly preinvex on $S$ if for all $x, y \in S$ such that $f(x) \neq f(y)$, and for any $\alpha \in(0,1)$, one has

$$
F(y+\alpha \eta(x, y)) \in \alpha F(x)+(1-\alpha) F(y)-\operatorname{int} D
$$

(ii) $F: S \rightarrow Y$ is said to be $D$-strictly preinvex on $S$ if for all $x, y \in S$ such that $x \neq y$, and for any $\alpha \in(0,1)$, one has

$$
F(y+\alpha \eta(x, y)) \in \alpha F(x)+(1-\alpha) F(y)-\operatorname{int} D .
$$

In [2], Jeyakumar et al. introduced the $*$-lower semicontinuity for vector-valued function as follows. 
Definition 1.5. The vector-valued function $F: S \rightarrow Y$ is $*$-lower semicontinuous if for every $q \in D^{*}, q(F)(\cdot)=\langle q, F(\cdot)\rangle$ is lower semicontinuous on $S$.

We will introduce a new notation as follows.

Definition 1.6. The vector-valued function $F: S \rightarrow Y$ is called $*$-upper semicontinuous if for every $q \in D^{*}, q(F)(\cdot)$ is upper semicontinuous on $S$.

Mohan and Neogy [4] introduced Condition C defined as follows.

Condition $C$. The vector-valued function $\eta: X \times X \rightarrow X$ is said to satisfy Condition $\mathrm{C}$ if for all $x, y \in X$ and for all $\alpha \in(0,1)$,

$$
\begin{gathered}
\eta(y, y+\alpha \eta(x, y))=-\alpha \eta(x, y), \\
\eta(x, y+\alpha \eta(x, y))=(1-\alpha) \eta(x, y) .
\end{gathered}
$$

And they proved that a differentiable function which is invex with respect to $\eta$ is also preinvex under Condition C. Mohan and Neogy also give an example which shows that Condition $\mathrm{C}$ may hold for a general class of function $\eta$, rather than just for the trivial case of $\eta(x, y)=x-y$.

In this paper, we will use the $*$-lower semicontinuity and $*$-upper semicontinuity to obtain some properties of $D$-preinvexity for vector-valued function in Section 2 and discuss the interrelations among $D$-preinvexity, $D$-semistrict preinvexity and $D$-strict preinvexity for vector-valued function in Section 3. The results in this paper generalize some results in $[5,8-10]$ from scalar case to vector case.

\section{Properties of the $D$-preinvex functions}

In this section, we will give some properties of $D$-preinvex functions.

Lemma 2.1. Let $S$ be a nonempty invex set in $X$ with respect to $\eta: X \times X \rightarrow X$, where $\eta$ satisfies Condition C. If $F: S \rightarrow Y$ satisfies the following conditions: for all $x, y \in S, F(y+$ $\eta(x, y)) \in F(x)-D$, and there exists an $\alpha \in(0,1)$ such that

$$
F(y+\alpha \eta(x, y)) \in \alpha F(x)+(1-\alpha) F(y)-D, \quad \forall x, y \in S
$$

then the set $A=\{\lambda \in[0,1] \mid F(y+\lambda \eta(x, y)) \in \lambda F(x)+(1-\lambda) F(y)-D\}$ is dense in the interval $[0,1]$.

Proof. Note that both $\lambda=0$ and 1 belong to set $A$ based on the fact that $F(y) \in F(y)-D$ and the assumption $F(y+\eta(x, y)) \in F(x)-D$. Suppose that the hypotheses hold and $A$ is not dense in $[0,1]$. Then there exist a $\lambda_{0} \in(0,1)$ and a neighborhood $N\left(\lambda_{0}\right)$ of $\lambda_{0}$ such that $N\left(\lambda_{0}\right) \cap A=\varnothing$. Define $\lambda_{1}=\inf \left\{\lambda \in A \mid \lambda \geq \lambda_{0}\right\}, \lambda_{2}=\sup \left\{\lambda \in A \mid \lambda \leq \lambda_{0}\right\}$, then we have $0 \leq \lambda_{2}<\lambda_{1} \leq 1$. Since $\{\alpha,(1-\alpha)\} \subset(0,1)$, we can choose $u_{1}, u_{2} \in A$ with $u_{1} \geq \lambda_{1}$ and $u_{2} \leq \lambda_{2}$ such that $\max \{\alpha,(1-\alpha)\}\left(u_{1}-u_{2}\right)<\lambda_{1}-\lambda_{2}$, then $u_{2} \leq \lambda_{2}<\lambda_{1} \leq u_{1}$. 
Next, let us consider $\bar{\lambda}=\alpha u_{1}+(1-\alpha) u_{2}$. From Condition C, we have

$$
\begin{aligned}
y+u_{2} & \eta(x, y)+\alpha \eta\left(y+u_{1} \eta(x, y), y+u_{2} \eta(x, y)\right) \\
& =y+u_{2} \eta(x, y)+\alpha \eta\left(y+u_{1} \eta(x, y), y+u_{1} \eta(x, y)-\left(u_{1}-u_{2}\right) \eta(x, y)\right) \\
& =y+u_{2} \eta(x, y)+\alpha \eta\left(y+u_{1} \eta(x, y), y+u_{1} \eta(x, y)+\frac{u_{1}-u_{2}}{u_{1}} \eta\left(y, y+u_{1} \eta(x, y)\right)\right) \\
& =y+u_{2} \eta(x, y)-\alpha \frac{u_{1}-u_{2}}{u_{1}} \eta\left(y, y+u_{1} \eta(x, y)\right) \\
& =y+\left(u_{2}+\alpha\left(u_{1}-u_{2}\right)\right) \eta(x, y)=y+\bar{\lambda} \eta(x, y), \quad \forall x, y \in S .
\end{aligned}
$$

Hence,

$$
\begin{aligned}
F(y+ & \bar{\lambda} \eta(x, y)) \\
& =F\left(y+u_{2} \eta(x, y)+\alpha \eta\left(y+u_{1} \eta(x, y), y+u_{2} \eta(x, y)\right)\right) \\
& \in \alpha F\left(y+u_{1} \eta(x, y)\right)+(1-\alpha) F\left(y+u_{2} \eta(x, y)\right)-D \\
& \subset \alpha\left[u_{1} F(x)+\left(1-u_{1}\right) F(y)-D\right]+(1-\alpha)\left[u_{2} F(x)+\left(1-u_{2}\right) F(y)-D\right]-D \\
& =\bar{\lambda} F(x)+(1-\bar{\lambda}) F(y)-D-D \subset \bar{\lambda} F(x)+(1-\bar{\lambda}) F(y)-D,
\end{aligned}
$$

that is, $\bar{\lambda} \in A$.

If $\bar{\lambda} \geq \lambda_{0}$, then $\bar{\lambda}-u_{2}=\alpha\left(u_{1}-u_{2}\right)<\lambda_{1}-\lambda_{2}$, and therefore $\bar{\lambda}<\lambda_{1}$. Because $\bar{\lambda} \geq \lambda_{0}$ and $\bar{\lambda} \in A$, this is a contradiction to the definition of $\lambda_{1}$. If $\bar{\lambda} \leq \lambda_{0}$, then $\bar{\lambda}-u_{1}=(1-\alpha)\left(u_{2}-\right.$ $\left.u_{1}\right)>\lambda_{2}-\lambda_{1}$, and therefore $\bar{\lambda}>\lambda_{2}$. Because $\bar{\lambda} \leq \lambda_{0}$ and $\bar{\lambda} \in A$, this is a contradiction to the definition of $\lambda_{2}$.

Theorem 2.2. Let $S$ be a nonempty open invex set in $X$ with respect to $\eta: X \times X \rightarrow X$, where $\eta$ satisfies Condition $C$, and $F: S \rightarrow Y$ is $*$-upper semicontinuous. If $F$ satisfies the following condition: for all $x, y \in S, F(y+\eta(x, y)) \in F(x)-D$, then $F$ is a D-preinvex function for the same $\eta$ on $S$ if and only if there exists an $\alpha \in(0,1)$ such that

$$
F(y+\alpha \eta(x, y)) \in \alpha F(x)+(1-\alpha) F(y)-D, \quad \forall x, y \in S .
$$

Proof. The necessity follows directly from the definition of $D$-preinvexity for the vectorvalued function $F$. We only need to prove the sufficiency.

Suppose that the hypotheses hold and $F$ is not $D$-preinvex on $S$. Then, there exist $x, y \in S$ and $\bar{\lambda} \in(0,1)$ such that

$$
F(y+\bar{\lambda} \eta(x, y)) \notin \bar{\lambda} F(x)+(1-\bar{\lambda}) F(y)-D .
$$

Let $z=y+\bar{\lambda} \eta(x, y)$. From Lemma 2.1, we know that there exists a sequence $\left\{\lambda_{n}\right\}$ with $\lambda_{n} \in A$ and $\lambda_{n}<\bar{\lambda}$ (the definition of $A$ in Lemma 2.1) such that $\lambda_{n} \rightarrow \bar{\lambda}(n \rightarrow \infty)$. Define 
$y_{n}=y+\left(\left(\bar{\lambda}-\lambda_{n}\right) /\left(1-\lambda_{n}\right)\right) \eta(x, y)$. Then $y_{n} \rightarrow y(n \rightarrow \infty)$. Note that $S$ is an open invex set with respect to $\eta$. Thus for $n$ is sufficiently large, we have $y_{n} \in S$.

Furthermore, by Condition C, we have

$$
y_{n}+\lambda_{n} \eta\left(x, y_{n}\right)=y+\left(\frac{\bar{\lambda}-\lambda_{n}}{1-\lambda_{n}}\right) \eta(x, y)+\lambda_{n} \eta\left(x, y+\left(\frac{\bar{\lambda}-\lambda_{n}}{1-\lambda_{n}}\right) \eta(x, y)\right)=y+\bar{\lambda} \eta(x, y)=z
$$

As $\lambda_{n} \in A$, we have

$$
F(z)=F(y+\bar{\lambda} \eta(x, y))=F\left(y_{n}+\lambda_{n} \eta\left(x, y_{n}\right)\right) \in \lambda_{n} F(x)+\left(1-\lambda_{n}\right) F\left(y_{n}\right)-D .
$$

By the $*$-upper semicontinuity of $F$ on $S$, for every $q \in D^{*}, q(F)(\cdot)$ is upper semicontinuous, it follows that for any $\varepsilon>0$, there exists an $N>0$ such that the following holds:

$$
q(F)\left(y_{n}\right) \leq q(F)(y)+\varepsilon, \quad \forall n>N .
$$

Hence,

$$
\begin{aligned}
q(F)(z) \leq & \lambda_{n} q(F)(x)+\left(1-\lambda_{n}\right) q(F)\left(y_{n}\right) \leq \lambda_{n} q(F)(x) \\
& +\left(1-\lambda_{n}\right)[q(F)(y)+\varepsilon] \longrightarrow \bar{\lambda} q(F)(x)+(1-\bar{\lambda})[q(F)(y)+\varepsilon] \quad(n \longrightarrow \infty) .
\end{aligned}
$$

Since $\varepsilon>0$ may be arbitrary small, then for all $q \in D^{*}$, we have

$$
q(F)(z) \leq \bar{\lambda} q(F)(x)+(1-\bar{\lambda}) q(F)(y) .
$$

Since $q$ is linear and by Lemma 1.1, we have

$$
F(z) \in \bar{\lambda} F(x)+(1-\bar{\lambda}) F(y)-D .
$$

Equation (2.11) is a contradiction to (2.5), thus the conclusion is correct.

Theorem 2.3. Let $S$ be a nonempty invex set in $X$ with respect to $\eta: X \times X \rightarrow X$, where $\eta$ satisfies Condition $C$, and $F: S \rightarrow Y$ is $*$-lower semicontinuous. If $F$ satisfies the following condition: for all $x, y \in S, F(y+\eta(x, y)) \in F(x)-D$, then $F$ is a D-preinvex function if and only if for all $x, y \in S$, there exists an $\alpha \in(0,1)$ such that

$$
F(y+\alpha \eta(x, y)) \in \alpha F(x)+(1-\alpha) F(y)-D .
$$

Proof. The necessity follows directly from the definition of $D$-preinvexity of $F$. We only need to prove the sufficiency.

Suppose that the hypotheses hold and $F$ is not $D$-preinvex on $S$. Then, there exist $x, y \in S$ and $\bar{\lambda} \in(0,1)$ such that

$$
F(y+\bar{\lambda} \eta(x, y)) \notin \bar{\lambda} F(x)+(1-\bar{\lambda}) F(y)-D .
$$

Let $x_{t}=y+t \eta(x, y), t \in(\bar{\lambda}, 1]$, and $B=\left\{x_{t} \in S \mid t \in(\bar{\lambda}, 1], F\left(x_{t}\right)=F(y+t \eta(x, y)) \in\right.$ $t F(x)+(1-t) F(y)-D\}, u=\inf \left\{t \in(\bar{\lambda}, 1] \mid x_{t} \in B\right\}$. It is easy to check that $x_{1} \in B$ from 
the assumption and $x_{\bar{\lambda}} \notin B$. Then, $t \in[\bar{\lambda}, u)$ implies $x_{t} \notin B$, and there exists a sequence $t_{n}$ with $t_{n} \geq u$ and $x_{t_{n}} \in B$ such that $t_{n} \rightarrow u(n \rightarrow \infty)$. Hence, $F\left(x_{t_{n}}\right)=F\left(y+t_{n} \eta(x, y)\right) \in$ $t_{n} F(x)+\left(1-t_{n}\right) F(y)-D$. Then for all $q \in D^{*}$, we have

$$
q(F)\left(x_{t_{n}}\right) \leq t_{n} q(F)(x)+\left(1-t_{n}\right) q(F)(y) .
$$

Since $F$ is $*$-lower semicontinous, for every $q \in D^{*}, q(F)(\cdot)$ is lower semicontinuous, it follows that

$$
\begin{aligned}
q(F)\left(x_{u}\right) & =q(F)(y+u \eta(x, y)) \leq \lim _{n \rightarrow \infty} q(F)\left(x_{t_{n}}\right) \\
& \leq \lim _{n \rightarrow \infty}\left[t_{n} q(F)(x)+\left(1-t_{n}\right) q(F)(y)\right]=u q(F)(x)+(1-u) q(F)(y) .
\end{aligned}
$$

Since $q$ is linear and by Lemma 1.1, we have $F\left(x_{u}\right) \in u F(x)+(1-u) F(y)-D$. Hence, $x_{u} \in B$.

Let $y_{t}=y+t \eta(x, y), t \in[0, \bar{\lambda})$, and $D=\left\{y_{t} \in S \mid t \in[0, \bar{\lambda}), F\left(y_{t}\right)=F(y+t \eta(x, y)) \in\right.$ $t F(x)+(1-t) F(y)-D\}, v=\sup \left\{t \in[0, \bar{\lambda}) \mid y_{t} \in D\right\}$. It is easy to check that $y_{0}=y \in D$ from the assumption and $y_{\bar{\lambda}}=y+\bar{\lambda} \eta(x, y) \notin D$. Then, $t \in(v, \bar{\lambda}]$ implies $y_{t} \notin D$, and there exists a sequence $t_{n}$ with $t_{n} \leq v$ and $y_{t_{n}} \in D$ such that $t_{n} \rightarrow v(n \rightarrow \infty)$. Hence $F\left(y_{t_{n}}\right)=$ $F\left(y+t_{n} \eta(x, y)\right) \in t_{n} F(x)+\left(1-t_{n}\right) F(y)-D$. Then for all $q \in D^{*}$, we have

$$
q(F)\left(y_{t_{n}}\right) \leq t_{n} q(F)(x)+\left(1-t_{n}\right) q(F)(y) .
$$

Since $F: S \rightarrow Y$ is $*$-lower semicontinous, for every $q \in D^{*}, q(F)(\cdot)$ is lower semicontinuous, it follows that

$$
\begin{aligned}
q(F)\left(y_{v}\right) & =q(F)(y+v \eta(x, y)) \leq \lim _{n \rightarrow \infty} q(F)\left(y_{t_{n}}\right) \\
& \leq \lim _{n \rightarrow \infty}\left[t_{n} q(F)(x)+\left(1-t_{n}\right) q(F)(y)\right]=v q(F)(x)+(1-v) q(F)(y) .
\end{aligned}
$$

Since $q$ is linear and by Lemma 1.1, we have $F\left(y_{v}\right) \in v F(x)+(1-v) F(y)-D$. Hence, $y_{v} \in D$.

By the definition of $u, v$, we have $0 \leq v<\bar{\lambda}<u \leq 1$. From Condition $\mathrm{C}$, for all $\lambda \in(0,1)$, we have

$$
\begin{aligned}
x_{u}+\lambda & \eta\left(y_{v}, x_{u}\right) \\
& =y+u \eta(x, y)+\lambda \eta(y+v \eta(x, y), y+u \eta(x, y)) \\
& =y+u \eta(x, y)+\lambda \eta(y+v \eta(x, y), y+v \eta(x, y)+(u-v) \eta(x, y)) \\
& =y+u \eta(x, y)+\lambda \eta\left(y+v \eta(x, y), y+v \eta(x, y)+\frac{u-v}{1-v} \eta(x, y+v \eta(x, y))\right) \\
& =y+u \eta(x, y)-\lambda \frac{u-v}{1-v} \eta(x, \eta(x, y+v \eta(x, y))) \\
& =y+[u-\lambda(u-v)] \eta(x, y)=y+[\lambda v+(1-\lambda) u] \eta(x, y) .
\end{aligned}
$$


From above, we get

$$
\begin{aligned}
\lambda F\left(y_{v}\right)+(1-\lambda) F\left(x_{u}\right) & \in \lambda[v F(x)+(1-v) F(y)-D]+(1-\lambda)[u F(x)+(1-u) F(y)-D] \\
& =[\lambda v+(1-\lambda) u] F(x)+[1-\lambda v-(1-\lambda) u] F(y)-D .
\end{aligned}
$$

Hence,

$$
\lambda F\left(y_{v}\right)+(1-\lambda) F\left(x_{u}\right)-D \subset[\lambda v+(1-\lambda) u] F(x)+[1-\lambda v-(1-\lambda) u] F(y)-D
$$

By the definition of $u, v$, we have

$$
\begin{aligned}
F\left(x_{u}+\lambda \eta\left(y_{v}, x_{u}\right)\right) & =F(y+[\lambda v+(1-\lambda) u] \eta(x, y)) \\
& \notin[\lambda v+(1-\lambda) u] F(x)+[1-\lambda v-(1-\lambda) u] F(y)-D .
\end{aligned}
$$

Hence, for all $\lambda \in(0,1)$,

$$
F\left(x_{u}+\lambda \eta\left(y_{v}, x_{u}\right)\right) \notin \lambda F\left(y_{v}\right)+(1-\lambda) F\left(x_{u}\right)-D .
$$

Equation (2.22) is a contradiction to (2.12), thus the conclusion is correct.

Remark 2.4. Theorems 2.2 and 2.3 generalize [9, Theorems 3.1 and 3.2] from scalar case to vector-valued case, respectively.

\section{Relationship among $D$-preinvexity, $D$-strict preinvexity, and $D$-semistrict preinvexity}

It is easy to see that $D$-strict preinvexity implies $D$-semistrict preinvexity by Definition 1.4 . The following examples illustrate that a $D$-semistrictly preinvex function may be neither a $D$-preinvex function nor a $D$-strictly preinvex function and a $D$-preinvex function does not imply a $D$-semistrictly preinvex function.

Example 3.1. This example illustrates that a semistrictly $D$-preinvex mapping may be neither a $D$-preinvex function nor a $D$-strictly preinvex function. Let $D=\{(x, y) \mid x \geq$ $0, y \geq 0\}, F(x)=\left(f_{1}(x), f_{2}(x)\right)$.

$$
\begin{gathered}
f_{1}(x)=\left\{\begin{array}{ll}
-|x| & \text { if }|x| \leq 1, \\
-1 & \text { if }|x| \geq 1,
\end{array} \quad f_{2}(x)= \begin{cases}-3|x| & \text { if }|x| \leq 1, \\
-3 & \text { if }|x| \geq 1,\end{cases} \right. \\
\eta(x, y)= \begin{cases}x-y & \text { if } x \geq 0, y \geq 0, \text { or } x \leq 0, y \leq 0, \\
x-y & \text { if } x>1, y<-1, \text { or } x<-1, y>1, \\
y-x & \text { if }-1 \leq x \leq 0, y \geq 0, \text { or }-1 \leq y \leq 0, x \geq 0, \\
y-x & \text { if } 0 \leq x \leq 1, y \leq 0, \text { or } 0 \leq y \leq 1, x \leq 0 .\end{cases}
\end{gathered}
$$


Then, $F$ is a semistrictly $D$-preinvex mapping on $S=R^{2}$ with respect to $\eta$. However, by letting $x=3, y=-3, \lambda=1 / 2$, we have

$$
\begin{gathered}
F(y+\lambda \eta(x, y))=F\left(-3+\frac{1}{2} \eta(3,-3)\right)=F(0)=(0,0), \\
\lambda F(x)+(1-\lambda) F(y)=F(3)=F(-3)=(-1,-3) .
\end{gathered}
$$

So

$$
\begin{gathered}
F(y+\lambda \eta(x, y)) \notin \lambda F(x)+(1-\lambda) F(y)-D, \\
F(y+\lambda \eta(x, y)) \notin \lambda F(x)+(1-\lambda) F(y)-\operatorname{int} D .
\end{gathered}
$$

That is, may be neither a $D$-preinvex function nor a $D$-strictly preinvex function with respect to the same $\eta$.

Example 3.2. This example illustrates that a $D$-preinvex mapping is not necessarily a $D$ semistrictly preinvex mapping. Let $D=\{(x, y) \mid x \geq 0, y \geq 0\}, F(x)=\left(f_{1}(x), f_{2}(x)\right)$,

$$
\begin{gathered}
f_{1}(x)=-|x|, \quad f_{2}(x)=-2|x| . \\
\eta(x, y)= \begin{cases}x-y & \text { if } x \geq 0, y \geq 0, \\
x-y & \text { if } x \leq 0, y \leq 0, \\
y-x & \text { if } x \leq 0, y \geq 0, \\
y-x & \text { if } x \geq 0, y \leq 0 .\end{cases}
\end{gathered}
$$

Then, $F$ is a $D$-preinvex mapping with respect to $\eta$ on $S=R^{2}$. However, by letting $y=1$, $x=2, \lambda=1 / 2$, we have $F(y)=F(1)=(-1,-2) \neq(-2,-4)=F(x)$, and

$$
\begin{aligned}
F(y+\lambda \eta(x, y)) & =F\left(1+\frac{1}{2} \eta(2,1)\right)=F\left(\frac{3}{2}\right)=\left(-\frac{3}{2},-3\right) \\
& =\frac{1}{2} F(2)+\frac{1}{2} F(1)=\lambda F(x)+(1-\lambda) F(y) .
\end{aligned}
$$

So

$$
F(y+\lambda \eta(x, y)) \notin \lambda F(x)+(1-\lambda) F(y)-\operatorname{int} D
$$

That is, $F$ is not a semistrictly $D$-preinvex mapping with respect to the same $\eta$.

About relationship between $D$-preinvexity and $D$-strict preinvexity, we have the following result.

Theorem 3.3. Let $S$ be a nonempty invex set in $X$ with respect to $\eta: X \times X \rightarrow X$, where $\eta$ satisfies Condition $C$, and $F: S \rightarrow Y$ is a $D$-preinvex function for the same $\eta$ on $S$. If $F$ satisfies the following condition: there exists an $\alpha \in(0,1)$ such that for all $x, y \in S$ with $x \neq y$ 
implying that

$$
F(y+\alpha \eta(x, y)) \in \alpha F(x)+(1-\alpha) F(y)-\operatorname{int} D,
$$

then $F$ is a D-strictly preinvex function on $S$.

Proof. Assume that $F$ is not a $D$-strictly preinvex function, then there exist $x, y \in S$ with $x \neq y$ and there exists $\lambda \in(0,1)$ such that

$$
F(y+\lambda \eta(x, y)) \notin \lambda F(x)+(1-\lambda) F(y)-\operatorname{int} D .
$$

Choose $\beta_{1}, \beta_{2}$ with $0<\beta_{1}<\beta_{2}<1$ and $\lambda=\alpha \beta_{1}+(1-\alpha) \beta_{2}$. Let $\bar{x}=y+\beta_{1} \eta(x, y), \bar{y}=$ $y+\beta_{2} \eta(x, y)$. Since $F$ is a $D$-preinvex function, we have

$$
F(\bar{x}) \in \beta_{1} F(x)+\left(1-\beta_{1}\right) F(y)-D, \quad F(\bar{y}) \in \beta_{2} F(x)+\left(1-\beta_{2}\right) F(y)-D .
$$

By Condition $\mathrm{C}$, we have

$$
\begin{aligned}
\bar{y}+\alpha & \eta(\bar{x}, \bar{y}) \\
& =y+\beta_{2} \eta(x, y)+\alpha \eta\left(y+\beta_{1} \eta(x, y), y+\beta_{1} \eta(x, y)+\left(\beta_{2}-\beta_{1}\right) \eta(x, y)\right) \\
& =y+\beta_{2} \eta(x, y)+\alpha \eta\left(y+\beta_{1} \eta(x, y), y+\beta_{1} \eta(x, y)+\frac{\left(\beta_{2}-\beta_{1}\right)}{1-\beta_{1}} \eta\left(x, y+\beta_{1} \eta(x, y)\right)\right) \\
& =y+\beta_{2} \eta(x, y)-\alpha \frac{\left(\beta_{2}-\beta_{1}\right)}{1-\beta_{1}} \eta\left(x, y+\beta_{1} \eta(x, y)\right) \\
& =y+\left(\beta_{2}-\alpha\left(\beta_{2}-\beta_{1}\right)\right) \eta(x, y)=y+\lambda \eta(x, y) .
\end{aligned}
$$

That is, $\bar{y}+\alpha \eta(\bar{x}, \bar{y})=y+\lambda \eta(x, y)$. By (3.7), we have

$$
F(y+\lambda \eta(x, y)) \in \alpha F(\bar{x})+(1-\alpha) F(\bar{y})-\operatorname{int} D .
$$

By (3.9), (3.11), and $D+\operatorname{int} D \subset \operatorname{int} D$, we have

$$
\begin{aligned}
F(y)+ & \lambda \eta(x, y) \\
\in & \alpha\left[\beta_{1} F(x)+\left(1-\beta_{1}\right) F(y)-D\right] \\
& +(1-\alpha)\left[\beta_{2} F(x)+\left(1-\beta_{2}\right) F(y)-D\right]-\operatorname{int} D \\
\subset & \left(\alpha \beta_{1}+(1-\alpha) \beta_{2}\right) F(x)+\left(1-\alpha \beta_{1}-(1-\alpha) \beta_{2}\right) F(y)-\operatorname{int} D \\
= & \lambda F(x)+(1-\lambda) F(y)-\operatorname{int} D .
\end{aligned}
$$

This is a contradiction to (3.8), hence $F$ is a $D$-strictly preinvex function on $S$.

Remark 3.4. If the vector-valued function $F: S \rightarrow Y$ is replaced by a scalar function $F$ : $S \rightarrow \mathbb{R}$ and $D=\{r \geq 0: r \in \mathbb{R}\}$, then by Theorem 3.5, we can obtain the following result, which is [5, Theorem 1]. 
Let $S$ be a nonempty invex set in $X$ with respect to $\eta: X \times X \rightarrow X$, where $\eta$ satisfies Condition $C$, and $f: S \rightarrow \mathbb{R}$ is a preinvex function for the same $\eta$ on $S$. If $f$ satisfies the following condition: there exists an $\alpha \in(0,1)$ such that for all $x, y \in S$ with $x \neq y$ implying that

$$
f(y+\alpha \eta(x, y)) \leq \alpha f(x)+(1-\alpha) f(y),
$$

then $f$ is a strictly preinvex function on $S$.

About relationship between $D$-semistrict preinvexity and $D$-strict preinvexity, we have a result as follows.

Theorem 3.5. Let $S$ be a nonempty invex set in $X$ with respect to $\eta: X \times X \rightarrow X$, where $\eta$ satisfies Condition $C$, and $F: S \rightarrow Y$ is a D-semistrictly preinvex function for the same $\eta$ on $S$. If $F$ satisfies the following condition: there exists an $\alpha \in(0,1)$ such that for all $x, y \in S$ with $x \neq y$ implying that

$$
F(y+\alpha \eta(x, y)) \in \alpha F(x)+(1-\alpha) F(y)-\operatorname{int} D
$$

then $F$ is a D-strictly preinvex function on $S$.

Proof. Since $F$ is $D$-semistrictly preinvex function, we only show that $F(x)=F(y), x \neq y$ implies that

$$
F(y+\lambda \eta(x, y)) \in \lambda F(x)+(1-\lambda) F(y)-\operatorname{int} D=F(x)-\operatorname{int} D, \quad \forall \lambda \in(0,1) .
$$

Let $\bar{x}=y+\alpha \eta(x, y)$. From (3.14) and for each $x, y \in S, x \neq y$, we have

$$
F(\bar{x})=F(y+\alpha \eta(x, y)) \in \alpha F(x)+(1-\alpha) F(y)-\operatorname{int} D=F(x)-\operatorname{int} D .
$$

For each $\lambda \in(0,1)$, if $\lambda<\alpha$, taking $u=(\alpha-\lambda) / \alpha$, then $u \in(0,1)$, and from Condition $\mathrm{C}$, we have $\bar{x}+u \eta(y, \bar{x})=y+\alpha \eta(x, y)+((\alpha-\lambda) / \alpha) \eta(y, y+\alpha \eta(x, y))=y+\alpha \eta(x, y)-(\alpha-$ $\lambda) \eta(x, y)=y+\lambda \eta(x, y)$. By the $D$-semistrictly preinvexity of $F$ and (3.16),

$$
\begin{aligned}
F(y+\lambda \eta(x, y)) & =F(\bar{x}+u \eta(y, \bar{x})) \in u F(\bar{x})+(1-u) F(y)-\operatorname{int} D \\
& \subset u(F(x)-\operatorname{int} D)+(1-u) F(y)-\operatorname{int} D \\
& =F(x)-\operatorname{int} D-\operatorname{int} D=F(x)-\operatorname{int} D .
\end{aligned}
$$

If $\lambda>\alpha$, taking $v=(\lambda-\alpha) /(1-\alpha)$, then $v \in(0,1)$ and from Condition $\mathrm{C}$, we have

$$
\begin{aligned}
\bar{x}+v \eta(x, \bar{x}) & =y+\alpha \eta(x, y)+\frac{\lambda-\alpha}{1-\alpha} \eta(x, y+\alpha \eta(x, y)) \\
& =y+\alpha \eta(x, y)+(\lambda-\alpha) \eta(x, y)=y+\lambda \eta(x, y) .
\end{aligned}
$$


From the $D$-semistrictly preinvexity of $F$ and (3.16),

$$
\begin{aligned}
F(y+\lambda \eta(x, y)) & =F(\bar{x}+v \eta(x, \bar{x})) \in v F(\bar{x})+(1-v) F(x)-\operatorname{int} D \\
& \subset v(F(x)-\operatorname{int} D)+(1-v) F(x)-\operatorname{int} D \\
& =F(x)-\operatorname{int} D-\operatorname{int} D=F(x)-\operatorname{int} D .
\end{aligned}
$$

This completes the proof.

Remark 3.6. Theorem 3.5 is the generalization of [8, Theorem 7].

About the relation between $D$-preinvexity and $D$-semistrict preinvexity, we will use the separation theorem of convex sets to prove the following result.

Theorem 3.7. Let $S$ be a nonempty invex set in $X$ with respect to $\eta: X \times X \rightarrow X$, where $\eta$ satisfies Condition $C$, and let $F: S \rightarrow Y$ be $*$-lower semicontinuous and D-semistrictly preinvex for the same $\eta$ on $S$. Then $F$ is a D-preinvex function on $S$.

Proof. Let $x, y \in S$. If $F(x) \neq F(y)$, then by the $D$-semistrict preinvexity of $F$, we have

$$
F(y+\lambda \eta(x, y)) \in \lambda F(x)+(1-\lambda) F(y)-\operatorname{int} D \subset \lambda F(x)+(1-\lambda) F(y)-D, \quad \forall \lambda \in(0,1) .
$$

If $F(x)=F(y)$, to show that $F$ is a $D$-preinvex function, we need to show that

$$
F(y+\lambda \eta(x, y)) \in F(x)-D, \quad \forall \lambda \in(0,1) .
$$

By contradiction, suppose there exists an $\alpha \in(0,1)$ such that

$$
F(y+\alpha \eta(x, y)) \notin F(x)-D
$$

Let $z_{\alpha}=y+\alpha \eta(x, y)$. Since $F(x)-D$ is a closed convex set, by the strong separation theorem for convex sets, there exist $0 \neq q \in Y^{*}$ and $b \in \mathbb{R}$ such that

$$
q(F)\left(z_{\alpha}\right)>b \geq q[(F)(x)-d], \quad \forall d \in D .
$$

Since $D$ is a cone, we have that $q(d) \geq 0$, for all $d \in D$, which implies that $q \in D^{*}$. By $0 \in D$ and (3.23), we have

$$
q(F)\left(z_{\alpha}\right)>q(F)(x)
$$

Since $\mathrm{F}$ is $*$-lower semicontinuous, there exists $\beta: \alpha<\beta<1$ such that

$$
q(F)\left(z_{\beta}\right)=q(F)(y+\beta \eta(x, y))>q(F)(x)=q(F)(y) .
$$

From Condition C,

$$
z_{\beta}=z_{\alpha}+\frac{\beta-\alpha}{1-\alpha} \eta\left(x, z_{\alpha}\right) .
$$


Hence, by (3.22) and $D$-semistrict preinvexity of $F$, we have

$$
F\left(z_{\beta}\right) \in \frac{\beta-\alpha}{1-\alpha} F(x)+\left(1-\frac{\beta-\alpha}{1-\alpha}\right) F\left(z_{\alpha}\right)-\operatorname{int} D .
$$

Since $q \in D^{*},(3.24)$ and (3.27) imply

$$
q(F)\left(z_{\beta}\right)<\frac{\beta-\alpha}{1-\alpha} q(F)(x)+\left(1-\frac{\beta-\alpha}{1-\alpha}\right) q(F)\left(z_{\alpha}\right)<q(F)\left(z_{\alpha}\right) .
$$

On the other hand, from Condition C,

$$
z_{\alpha}=z_{\beta}+\left(1-\frac{\alpha}{\beta}\right) \eta\left(y, z_{\beta}\right)
$$

Therefore, by (3.25) and $D$-semistrict preinvexity of $F$, we have

$$
F\left(z_{\alpha}\right) \in\left(1-\frac{\alpha}{\beta}\right) F(y)+\frac{\alpha}{\beta} F\left(z_{\beta}\right)-\operatorname{int} D .
$$

Since $q \in D^{*},(3.25)$ and (3.30) imply

$$
q(F)\left(z_{\alpha}\right)<\left(1-\frac{\alpha}{\beta}\right) q(F)(y)+\frac{\alpha}{\beta} q(F)\left(z_{\beta}\right)<q(F)\left(z_{\beta}\right),
$$

which contradicts (3.28). This complete the proof.

Remark 3.8. Theorem 3.7 is a generalization of [10, Theorem 5.1].

Theorem 3.9. Let $S$ be a nonempty invex set in $X$ with respect to $\eta: X \times X \rightarrow X$, where $\eta$ satisfies Condition $C$, and $F: S \rightarrow Y$ is a D-preinvex function for the same $\eta$ on $S$. If for every $x, y \in S, F(x) \neq F(y)$, there exists an $\alpha \in(0,1)$ such that

$$
F(y+\alpha \eta(x, y)) \in \alpha F(x)+(1-\alpha) F(y)-\operatorname{int} D,
$$

then $F$ is a D-semistrictly preinvex function on $S$.

Proof. For each $x, y \in S$ satisfy $F(x) \neq F(y)$ and $\lambda \in(0,1)$, by assumption, we have

$$
F(y+\lambda \eta(x, y)) \in \lambda F(x)+(1-\lambda) F(y)-D .
$$

If $\lambda \leq \alpha$, from Condition $\mathrm{C}$,

$$
\begin{aligned}
y+\frac{\lambda}{\alpha} \eta(y+\alpha \eta(x, y), y) & =y+\frac{\lambda}{\alpha} \eta(y+\alpha \eta(x, y), y+\alpha \eta(x, y)-\alpha \eta(x, y)) \\
& =y+\frac{\lambda}{\alpha} \eta(y+\alpha \eta(x, y), y+\alpha \eta(x, y)+\eta(y, y+\alpha \eta(x, y))) \\
& =y-\frac{\lambda}{\alpha} \eta(y, y+\alpha \eta(x, y))=y+\lambda \eta(x, y) .
\end{aligned}
$$


According to (3.32) and (3.33), we have

$$
\begin{aligned}
F(y+\lambda \eta(x, y)) & =F\left(y+\frac{\lambda}{\alpha} \eta(y+\alpha \eta(x, y), y)\right) \\
& \in \frac{\lambda}{\alpha} F(y+\alpha \eta(x, y))+\left(1-\frac{\lambda}{\alpha}\right) F(y)-D \\
& \subset \frac{\lambda}{\alpha}[\alpha F(x)+(1-\alpha) F(y)-\operatorname{int} D]+\left(1-\frac{\lambda}{\alpha}\right) F(y)-D \\
& \subset \lambda F(x)+(1-\lambda) F(y)-\operatorname{int} D .
\end{aligned}
$$

If $\lambda>\alpha$,

$$
0<\frac{1-\lambda}{1-\alpha}<1
$$

From Condition C,

$$
y+\alpha \eta(x, y)+\left(1-\frac{1-\lambda}{1-\alpha}\right) \eta(x, y+\alpha \eta(x, y))=y+\lambda \eta(x, y) .
$$

According to (3.32) and (3.33), we have

$$
\begin{aligned}
F(y+\lambda \eta(x, y)) & =F\left(y+\alpha \eta(x, y)+\left(1-\frac{1-\lambda}{1-\alpha}\right) \eta(x, y+\alpha \eta(x, y))\right) \\
& \in \frac{1-\lambda}{1-\alpha} F(y+\alpha \eta(x, y))+\left(1-\frac{1-\lambda}{1-\alpha}\right) F(x)-D \\
& \subset \frac{1-\lambda}{1-\alpha}[\alpha F(x)+(1-\alpha) F(y)-\operatorname{int} D]+\left(1-\frac{1-\lambda}{1-\alpha}\right) F(x)-D \\
& \subset \lambda F(x)+(1-\lambda) F(y)-\operatorname{int} D .
\end{aligned}
$$

Equations (3.35) and (3.38) imply that $F$ is a $D$-semistrictly preinvex function on $S$.

Remark 3.10. Theorem 3.9 is a new result even in scalar case.

Remark 3.11. It is yet unclear whether there exist similar results with those in this paper while the single-valued function $F: S \rightarrow Y$ replaced by a set-valued function $F: S \rightarrow 2^{Y}$.

\section{Conclusions}

In this paper, we firstly obtain two properties of $D$-preinvexity for vector-valued function which are equivalent conditions in terms of the $D$-preinvexity and intermediate-point $D$-preinvexity. We then get two sufficient conditions of the $D$-strict preinvexity in terms of intermediate-point $D$-strict preinvexity and $D$-preinvexity (or $D$-semistrict preinvexity). We finally obtain both the sufficient condition and necessary condition of the $D$ preinvexity in terms of the $D$-semistrict preinvexity. 


\section{Acknowledgments}

This paper was supported by the National Natural Science Foundation of China (Grant no. 70432001 and Grant no. 10471159), the Science and Technology Research Project of Chinese Ministry of Education (no. 206123), and the Postdoctoral Science Foundation of China (no. 2005038133). The authors would like to express their thanks to the referees for helpful suggestions.

\section{References}

[1] D. Bhatia and A. Mehra, Lagrangian duality for preinvex set-valued functions, Journal of Mathematical Analysis and Applications 214 (1997), no. 2, 599-612.

[2] V. Jeyakumar, W. Oettli, and M. Natividad, A solvability theorem for a class of quasiconvex mappings with applications to optimization, Journal of Mathematical Analysis and Applications $\mathbf{1 7 9}$ (1993), no. 2, 537-546.

[3] K. R. Kazmi, Some remarks on vector optimization problems, Journal of Optimization Theory and Applications 96 (1998), no. 1, 133-138.

[4] S. R. Mohan and S. K. Neogy, On invex sets and preinvex functions, Journal of Mathematical Analysis and Applications 189 (1995), no. 3, 901-908.

[5] J.-W. Peng and X. M. Yang, Two properties of strictly preinvex functions, Operations Research Transactions 9 (2005), no. 1, 37-42.

[6] T. Weir and V. Jeyakumar, A class of nonconvex functions and mathematical programming, Bulletin of the Australian Mathematical Society 38 (1988), no. 2, 177-189.

[7] T. Weir and B. Mond, Pre-invex functions in multiple objective optimization, Journal of Mathematical Analysis and Applications 136 (1988), no. 1, 29-38.

[8] X. M. Yang, Semistrictly convex functions, Opsearch 31 (1994), no. 1, 15-27.

[9] X. M. Yang and D. Li, On properties of preinvex functions, Journal of Mathematical Analysis and Applications 256 (2001), no. 1, 229-241.

[10] Semistrictly preinvex functions, Journal of Mathematical Analysis and Applications 258 (2001), no. 1, 287-308.

[11] X. M. Yang, X. Q. Yang, and K. L. Teo, Explicitly B-preinvex functions, Journal of Computational and Applied Mathematics 146 (2002), no. 1, 25-36.

Jian-Wen Peng: Department of Management Science, School of Management, Fudan University, Shanghai 200433, China

Current address: College of Mathematics and Computer Science, Chongqing Normal University, Chongqing 400047, China

E-mail address: jwpeng6@yahoo.com.cn

Dao-Li Zhu: Department of Management Science, School of Management, Fudan University,

Shanghai 200433, China

E-mail address: dlzhu@fudan.edu.cn 